Voix et Images

voixetimages

\title{
Par les sentiers battus
}

\section{François Dumont}

Volume 19, numéro 3 (57), printemps 1994

Science et fiction au Québec : L’émergence d'un savoir

URI : https://id.erudit.org/iderudit/201128ar

DOI : https://doi.org/10.7202/201128ar

Aller au sommaire du numéro

\section{Éditeur(s)}

Université du Québec à Montréal

\section{ISSN}

0318-9201 (imprimé)

1705-933X (numérique)

Découvrir la revue

\section{Citer cet article}

Dumont, F. (1994). Par les sentiers battus. Voix et Images, 19(3), 658-665.

https://doi.org/10.7202/201128ar d'utilisation que vous pouvez consulter en ligne.

https://apropos.erudit.org/fr/usagers/politique-dutilisation/ 


\section{Poésie}

\section{Par les sentiers battus}

\section{François Dumont, Université du Québec à Trois-Rivières}

S'éloignant tout autant de la tradition du nouveau que du recyclage parodique, plusieurs poètes québécois contemporains renouent respectueusement avec d'anciennes esthétiques. Les polémiques sur l'avant-garde, étroitement associées à la poésie il n'y a pas si longtemps, paraissent déjà désuètes: il s'agirait maintenant, semble-t-il, de s'inscrire dans une continuité historique.

Parmi les recueils récents qui participent de cette tendance, il faut d'abord signaler une très belle réussite: L'Avant-printemps à Montréal, de Robert Melançon ${ }^{1}$. Jusqu'à tout récemment, ainsi que je l'indiquais dans la précédente chronique, la poésie de Melançon gravitait autour du livre Peinture aveugle ${ }^{2}$. Mais il semble que les marges de ce projet aient tendance à s'élargir considérablement. En effet, après avoir proposé $A u$ petit matin ${ }^{3}$, écrit en collaboration avec Jacques Brault, Melançon a recueilli divers poèmes relativement disparates, dont certains ont déjà paru en revue. Alors que la cohérence de Peinture aveugle était fondée sur un projet, les poèmes de L'Avant-printemps à Montréal n'auraient trouvé leur cohésion que dans l'assemblage (c'est du moins ma lecture de l'exergue de Saint-Denys Garneau: "Le noud s'est mis à sentir/Les tours de corde dont il est fait ").

L'autonomie des textes ne fait pas de ce livre un regroupement arbitraire notamment parce que le travail du vers régulier y est souvent à l'honneur. Le poème éponyme transforme d'entrée de jeu l'octosyllabe du titre en une sorte de la formel. Les procédés d'enjambement rappellent le vers libre de Peinture aveugle, mais on entend constamment aussi divers échos, par exemple dans ces strophes carrées très verlainiennes :

Le soleil couchant, Le bruit des voitures, Et ces gens qui vont Partout à la fois : 
Tout ça plaît, tel quel!

[...]

On va, enivré

Par la multitude,

Et les lampadaires

Parsèment d'étoiles

Les rues qui se couchent (p. 29).

La tonalité de ce poème et de plusieurs autres rappelle celle du recueil $A u$ petit matin, bien que les contraintes orientales aient cédé le pas à la tradition occidentale. Dans la poésie de Melançon se trouve toujours une sorte de disponibilité méditative; elle s'accompagne, ici, d'un grand souci du lecteur, qu'il soit dédicataire (certains poèmes adoptent la forme de la lettre intime) ou lecteur anonyme (qui est interpellé comme un "ami"). L'un des aspects de cette attention portée au lecteur est le poli des textes: si la banalité prend beaucoup de place dans ce livre, elle est toujours portée par un travail qui la rend lumineuse.

Dans les recueils de poésie, les exergues ont parfois l'air de décorations surajoutées, platement stratégiques. Ce n'est pas le cas dans Les Intrusions de l'œil suivi d'un Petit Traité de beauté, le plus. récent recueil de Célyne Fortin ${ }^{4}$. Une petite anthologie d'épigraphes témoigne éloquemment de la quête historique à laquelle je faisais allusion plus haut. Le point de départ de la première partie est donné par Pline l'Ancien. Il sera relayé par des hommes et des femmes de diverses époques de l'histoire occidentale qui se sont intéressés à la notion de beauté. Cette notion est d'abord considérée dans sa relation avec la vie des femmes. Un discours didactique prévisible invite à une mise en question des stéréotypes. Mais s'y ajoute une riche réflexion sur le. regard et sur le corps qui dépasse le didactisme.

La première partie, qui occupe les trois quarts de l'ouvrage, est fortement structurée. Sept sections reprennent le même tracé et les mêmes titres. Au début de chacune des sections, un collage de l'auteure représente un homme, dessiné très techniquement, et une femme, suggérée très vaporeusement. Un exergue pose ensuite, à son tour, un motif. Le premier poème constitue une description et un commentaire du collage; un second focalise sur la place de la femme dans le tableau, alors que les trois suivants, moins directement liés au tableau d'origine, concernent respectivement le dualisme du JE, la 
souffrance au féminin et la passion amoureuse. Le Petit Traité final, pour sa part, accorde une grande place à la citation et pose un regard quasi clinique sur les diktats relatifs à la beauté du corps.

Célyne Fortin a donc choisi, dans ce livre très composé, d'associer la poésie à l'image, ce qui s'avère très réussi, et au commentaire analytique, ce qui, à mon avis, l'est moins. Dans plusieurs poèmes, la succession de bonheurs d'expression et de vers explicatifs produit de curieux hiatus. Dans d'autres poèmes, cependant, la cohabitation de la poésie et du discours analytique est plus heureuse:

Elle est deux qui s'observe de l'extérieur.

Elle se voit personnage multiple

vivant le théâtre et la réalité.

Mais poursuivant son image de l'intérieur

elle se perçoit comme la demie de l'une

se plaçant au second centre des possibles (p. 37).

\section{$*^{*} *$}

Parmi tous les "sentiers battus " de la poésie du vingtième siècle, le surréalisme fut certainement l'un des plus fréquentés. Il ne l'est plus beaucoup aujourd'hui, sinon par quelques irréductibles qui, comme Jean Charlebois, continuent de cultiver la fantaisie et de célébrer le désir.

J'ai été très étonné du passage de Charlebois des Éditions du Noroît aux Éditions de l'Hexagone, avec la publication de Courps ${ }^{5}$. En faisant paraître pas moins de onze recueils au Noroît, Charlebois avait profondément marqué cette maison. Il y était vraiment à sa place, puisque l'audace graphique était partie prenante de sa poétique et que le Noroît était - et demeure - l'une des rares maisons d'édition de poésie sensible au caractère créatif du graphisme. À l'Hexagone, où la variété n'a plus rien à envier à celle que cultive depuis longtemps le Noroît, la présentation des livres est austère et offre peu de surprises, même si on a pris l'heureuse décision de remplacer les photographies d'auteurs par des œuvres d'art sur la page de couverture.

Dans ce contexte un peu hostile à sa manière, Jean Charlebois s'en est plutôt bien tiré, en réussissant à surprendre très simplement: les titres des poèmes ont été placés à la fin des textes, ce qui suffit à déstabiliser la lecture, le temps de quitter les réflexes coutumiers. De plus, des "notes et variantes" ont été ajoutées à la fin du recueil, ironi- 
sant sur l'esprit de sérieux auquel s'oppose tout à fait la poésie de Charlebois. Cela ne veut pas dire qu'elle soit sans conséquence; car le jeu de l'improvisation prend force d'éthique en s'opposant aux horreurs planifiées :

Les mains du coeur qui tremblent

la tête coupée

comme une fontaine éteinte

et le mortel

et la morale

les chiens qui nous dirigent

qui font la belle que font les beaux .

nous ne sommes pas des biens nécessaires (p. 133).

La poésie de Charlebois fait feu de tout bois, mais privilégie les ressources de la répétition et du jeu de mots. Les facilités ("ta notion du monde et ta lotion pour le corps", p. 28) et les clichés ("seul ensemble avec toin, p. 45) sont emportés par un souffle inventif qui a peu d'équivalents dans la poésie québécoise actuelle.

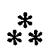

Il y a plus de trente ans que Wifrid Lemoine n'avait publié de poésie. À partir des années cinquante, son œuvre discrète s'était d'abord orientée vers la poésie, puis vers la prose narrative. Mais Lemoine s'est surtout imposé comme animateur à Radio-Canada. Il fut notamment un remarquable interviewer littéraire à la radio (pour s'en convaincre, il suffit de lire ses entretiens avec André Belleau dans la première partie de Notre Rabelais ${ }^{6}$ ).

Dans Passage à l'aube ${ }^{7}$, Lemoine renoue avec la poésie en privilégiant, sous l'égide d'Héraclite et de René Char, la forme de l'aphorisme. Il s'agit d'une forme difficile, menacée à la fois par l'obscurité et par le poncif. C'est surtout à cette deuxième pente que Passage à l'aube cède trop souvent, à mon avis, dans des énoncés comme "J'aimerais un jour voir s'éveiller/l'assassin de la mort " (p. 31), ou encore: "Quand je sors de mon rêve/je me bute à l'illusion" (p. 56).

Tout n'est pas de la même eau, cependant. À vrai dire, ce sont les textes qui s'éloignent le plus de l'aphorisme qui m'ont semblé les plus intéressants; notamment de petits poèmes plus développés qui, comme celui-ci, mettent en scène l'évanescence: 
Morte la fleur

Joie de mon regard

En étoile gourmande

Tige ployée

Couleurs passées

d'anciennes joies

Ou fatiguée peut-être

Gavée de lumière délavée

À bout de splendeurs

[...] (p. 44).

Mais les poèmes proprement dits sont peu nombreux, si bien que ce recueil, comme ensemble, ne réussit pas, de mon point de vue, à construire une véritable dynamique.

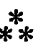

Daniel Dargis est, lui aussi, un poète discret. Son huitième recueil, Lumière artésienne ${ }^{8}$, qui ne paie pas de mine, risque de passer inaperçu. Ce serait dommage, car il s'agit d'un livre qui témoigne d'une authentique mise en question de la poésie - et qui n'oublie pas la part autocritique. Celle-ci aurait pu s'étendre à certains détails formels (allitérations malheureuses comme ajusqu'où la tourmente déroutet-elle", p. 28; formules contournées comme "la fuite ne pouvant désencombrer l'inaudible", p. 48). Malgré cela, le recueil dessine un trajet critique d'une acuité soutenue.

Le livre est divisé en cinq parties qui regroupent exclusivement des poèmes en vers libres. Il m'a semblé que ces cinq parties se ramenaient en fait à trois étapes principales: le désabusement, le détachement et le rapatriement. La critique fait donc place à un "livre béant", avant d'aboutir à une projection qui rappelle la poétique de Gaston Miron:

je touche le caillou éclairant le vide des placards

la pluie verte

les mots d'oiseaux plongés dans le vent

avec l'éloquence des vagues et des rochers

je prends les soleils qui nagent entre nous

j'entends où vous êtes

territoire qui s'épanche

par millions de ciels inédits

amenez-moi où tout commence

galaxies de mots

je me rapatrie (p. 56). 
Ce rapatriement est donc annoncé plutôt que réalisé. Il laisse attendre une poétique collective renouvelée qui puisse faire contrepoids à " la complainte moribonde de nos humiliations" (p. 55).

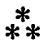

Claude Beausoleil fait paraître coup sur coup deux recueils: L'Usage du temps ${ }^{9}$ et Le Déchiffrement du monde ${ }^{10}$, qui se présentent ainsi comme une sorte de diptyque. Il s'agit de deux petits livres contrastés, d'intérêt inégál, qui participent néanmoins chacun à sa manière d'une mise en perspective historique.

Dans L'Usage du temps, une enfilade de quatrains compose un seul long poème. Lès vers tournent vaguement autour de l'alexandrin. En cela, ils sont emblématiques, car tout, dans ce recueil, paraît approximatif: autant le recours aux traditions prosodiques que le discours lui-même, qui confine à l'autocélébration redondante. La quatrième de couverture l'expose du reste clairement: il s'agirait d'un art poétique "où la poésie nous rappelle qu'elle est nécessité". De fait, elle ne cesse de rappeler qu'elle est à la fois une chose et son contraire:

oracle ou deuil souplement les détails

en arrivent à forcer l'adhésion (p. 7).

Où est la poésie? La poésie est partout; elle est début et fin, du moment qu'on "adhère "à cette proclamation d'une "souplesse" diffuse où tout s'annule,

jusqu'à laisser les choses devenir ce qu'elles

ont à être dans la perpétuelle respiration (p. 64).

Il en va tout autrement dans Le Déchiffrement du monde. Ici, la poésie se bute à des difficultés. Claude Beausoleil, qui a le souci d'entretenir la mémoire de la poésie québécoise en interrogeant des œuvres trop vite méprisées (celle de Louis Fréchette, par exemple) ou trop souvent négligées (celle de Clément Marchand, notamment), se situe ici, comme Daniel Dargis, dans le droit fil des préoccupations de Gaston Miron. En effet, la question de la langue, en regard de la collectivité et de la poésie, se trouve au cour du livre.

Dès la première page, Le Déchiffrement du monde prend le contre-pied de L'Usage du temps:

La langue est une enfance à garder en secret.

Une vaste zone enfouie, parfois fauve, 
Soụlève sa mémoire. Le noir profane,

De force emporte ses prophéties.

Il faut être fidèle, les jours sont démunis.

Vous vous dites cela,

Quand la loi vous affirme

Défendre vous ne savez trop quoi (p. 7).

"Oracle" et "deuil ", qui s'annulaient avec "souplesse" dans L'Usage $d u$ temps, reprennent ici leur force antithétique. La mémoire, pour s'opposer à la loi, ne peut plus "laisser les choses devenir". Elle est confrontée à

[...] tout ce qui empêche

L'éclat de la nécessité (p. 66).

Aussi Beausoleil renonce-t-il au halo poétique au profit d'une interrogation précise, qui convainc mieux de l'intérêt de la poésie que tous les éloges emphatiques.

$$
\text { ** }
$$

L'Oubli du monde, de Paul Bélanger ${ }^{11}$, dessine une patiente quête de la mémoire personnelle. L'enfance - par le biais, notamment, des figures du père et du grand-père - et les "amitiés perdues" sont évoquées dans l'espoir que le passé prenne un sens. Les rites religieux de l'enfance, désuets, sont remplacés par le poème, qui n'a que de très fragiles pouvoirs:

les matines sonnaient sur les fonts baptismaux

où ma première sœur fut admise dans le rituel

que je saisissais à peine

[...]

le poème demeure ma façon de prier

il porte la mémoire du monde

de notre aventure fascinante

et dérisoire (p. 18).

Cette poésie, on le voit, se montre très simple et très référentielle. Lorsqu'elle parle d'elle-même, c'est avec circonspection: comme la religion de l'enfance, elle s'impose et intrigue, sans illuminer. Mais puisque "sa lumière est projetée dans la durée" (p. 75), les moindres souvenirs sont précieusement consignés. On ne sait jamais :

ces quelques mots s'enquièrent de toi

s'ils te rejoignent j'aurai franchi

la frontière du temps

mais rien n'aura été expliqué (p. 76). 
Ce recueil dubitatif tient le lyrisme à distance, mais n'exclut pas l'émotion, par exemple lorsque la mort du grand-père est sobrement retrouvée. La poésie affleure et jamais Bélanger n'en rajoute: il tient le fil ténu du souvenir, sachant qu'il peut à tout moment se rompre.

"L'oubli du monde"? Dans un sens seulement: le monde oublie la poés ie, assurément; cependant la poésie d'aujourd'hui, de multiples manières, cultive sa mémoire.

1. Robert Melançon, L'Avant-printemps à Montréal, Montréal, VLB éditeur, 1994, 62 p.

2. Une édition revue et corrigée de Peinture aveugle (texte bilingue; traduction de Philip Stratford) a paru chez Vehicule Press (Montréal) en 1985.

3. Jacques Brault et Robert Melançon, Au petit matin, Montréal, l'Hexagone, coll. "Poésie *, 1993, [n.p.].

4. Célyne Fortin, Les Intrusions de l'œil suivi d'un Petit Traité de beauté, Montréal/ Paris, Le Noroît/Erti Éditeur, coll. "Résonance *, 1993, 85 p.

5. Jean Charlebois, Coeurps, Montréal, l'Hexagone, coll. *Poésie *, 1994, 166 p.

6. André Belleau, Notre Rabelais, Montréal, Boréal, coll. «Papiers collés*, 1990, p. 1585.

7. Wilfrid Lemoine, Passage à l'aube, Montréal, l'Hexagone, coll. «Poésie», 1994, $71 \mathrm{p}$.

8. Daniel Dargis, Lumière artésienne, Trois-Rivières, Écrits des Forges, 1993, $60 \mathrm{p}$.

9. Claude Beausoleil, L'Usage du temps, Montréal, Les Herbes rouges, 1993, 64 p.

10. Claude Beausoleil, Le Déchiffrement du monde, Montréal, Les Herbes rouges, 1993, 66 p.

11. Paul Bélanger, L'Oubli du monde, Montréal/Saint-Pierre-la-Vieille, Le Norôit/Atelier La Feugraie, coll. «Résonance», 1993, 77 p. 